\title{
THE TOMB-CHAPEL OF NEBAMUN AT THE BRITISH MUSEUM : THE DIGITAL, THE SPECTACULAR, AND THE INCONSPICUOUS UNDER ONE LIMESTONE ROOF
}

Francesca Monti

Department of Museum Studies

University College London

Institute of Archaeology

31-34 Gordon Square

London, WC1H OPY

United Kingdom

f.monti@ucl.ac.uk

Abstract - This article presents the evaluation of the digital resources for the Nebamun Gallery at the British Museum in the context of other display elements. The gallery exhibits eleven beautiful paintings from the ancient Egyptian tomb-chapel of Nebamun together with 180 objects and a computer simulation of the funerary chapel. The evaluation programme was designed drawing from the concepts and methods of space syntax, design idioms, beauty and usability, object biographies, and visual communication. The research uncovered a symbiotic interplay between exhibits which encourages visitors to view different sources of evidence and to draw connections between the objects, the paintings and the digital reconstruction.

\section{INTRODUCTION}

The dilemma of spectacular and inconspicuous objects in museums

At the heart of this research lies the assumption that the way something looks influences our behaviour towards it [1]. In simplified terms, museums objects fall into two categories: spectacular and inconspicuous (figure 1 and 2).
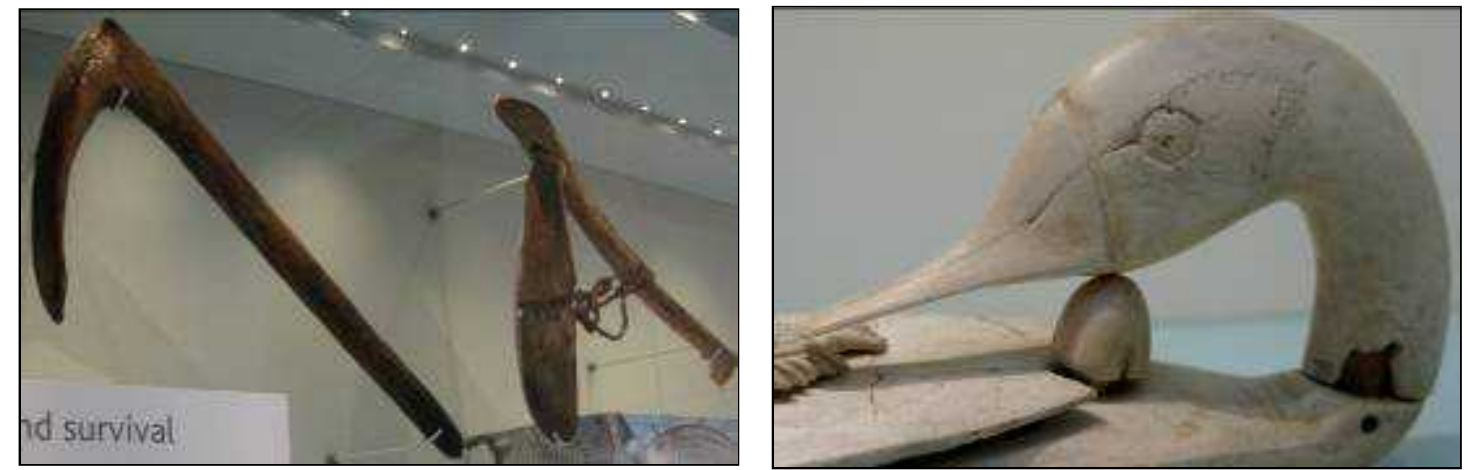

Figures 1 and 2. Inconspicuous wooden hoes (left) and a spectacular duck-shaped cosmetic box (right) from the tomb-chapel of Nebamun at the British Museum. 
Spectacular objects easily attract and hold the viewer. This may be because of their material qualities [2], such as colour, size, shape, symmetry, and fabric; and/or their non-physical attributes, such as age [2], iconicity, sense of past, magical significance [3], material value, and familiarity [4]. The latter qualities are nevertheless signalled by physical characteristics.

Inconspicuous objects have inner qualities and stories that are buried deeper within their appearance. Research indicates [e.g. 1,2,5,6,7] that the appearance of objects contributes to the formation of split-second assumptions that visitors consciously or involuntarily make about the depth and quality of the experience a specific object can provide. Because at a first glance they do not promise rewarding interactions, such objects are often neglected during museum visits unless an appropriate display and interpretation strategy is in place.

It came as a serendipitous revelation to observe that in the tomb-chapel of Nebamun at the British Museum inconspicuous objects and spectacular ones don't compete for visitor attention, but rather work together to convey the exhibition's messages. This anecdotal observation gathered during a family visit was the catalyst for an investigation of the reasons behind the apparent symbiotic relationship and, in particular, the role of the exhibition's digital resources in relation to other elements of display and interpretation.

\section{METHODOLOGY}

\section{Objectives and questions}

This study had two main objectives: to ascertain to what extent spectacular and inconspicuous objects can work together to deliver the exhibition messages rather than competing for visitor attention; and to establish the contribution of individual components in an exhibition, such as objects, computer interactives and simulations, which are the focus of this article. In addition, it was an opportunity to pilot an improved version of the author's methodology for assessing a museum gallery.

Specific questions addressed were: how do people view objects and how do they link them to create narratives? What is the role of videos in conveying such narratives? How do visitors receive a computer simulation in a British Museum permanent exhibition? Are the museum's intentions successfully conveyed to visitors?

\section{A tripartite and interdisciplinary evaluation framework}

The evaluation methodology stemmed from the premise that an exhibition is characterised by three key dimensions: the gallery; its reception among visitors; and the museum's creative forces. To understand an exhibition and to assess its success among audiences one needs to consider the three dimensions in combination.

It is also assumed that visitors' encounters with objects are forged by a series of factors and operate on many levels [1]. Broadly speaking, these variables can be grouped in three main areas of investigation: the visitor, the object, and the settings.

The three dimensions of an exhibition (the gallery, its reception among visitors, and the museum intervention) and the tripartite visitor-object-setting model informed the evaluation strategy. The methods employed were: 
- review of existing knowledge: existing visitor studies for Gallery 61 were examined and incorporated in the findings

- gallery audit and assessment of the video against check tables: check tables [5] which utilise the principles inherent in five selected concepts (see p.3) were employed to conduct a multi-perspective audit of the visit potential of the exhibition and to analyse the gallery digital reconstruction of the tomb-chapel

- assessment of the webpage against five criteria: the interactive webpage was appraised against five criteria developed during previous research [8]

- interview with museum staff: a semi-structured interview with the curator, interpretation officer, and designer was conducted to understand the museum's strategy and behind Nebamun Gallery and the dynamics which created it

- analysis of space and visitor circulation patterns: the results from visitor circulation studies and observations conducted by the British Museum [9] were employed to understand areas of interest and neglect within the gallery and to detect the most common patterns of interaction in relation to space, design, display elements and objects

- visitor survey: randomly selected visitors were interviewed after their viewing of the gallery to understand how they view, select, and make sense of gallery exhibits. Data from the British Museum gallery survey were also considered.

\section{Conceptual framework}

To comprehensively address the visitor-gallery-museum model, an investigation drawing on the disciplines of architecture, design, cognitive science, museology, material culture, and communication theories was conducted. The concepts of space syntax, design idioms, object biographies, beauty and usability, and visual communication were selected.

Space syntax is both the theory and the method employed in the analysis and description of a built environment [10]. Some of the notions and methods of space syntax were borrowed to explore strengths and weakness of the exhibition space, and to trace a correlation between attracting and holding power of specific exhibition features, spatial characteristics and visitor movement patterns.

In exhibitions design idioms - colour, light, text, and use of space - are employed to encourage circulation patterns, to elicit specific feelings, and to aid appreciation of the objects [11]. By looking at effective ways of dealing with these in an exhibition context one can address issues related to the attractive and holding powers of exhibits.

Drawing on the anthropological work on the cultural biography of things [12] the life trajectories of specific objects and the relationship they form with other objects and people can be used to understand how different individuals (for example audiences, curators, and collectors) encounter objects and make sense of them [13].

From the field of design came the notion that there is a need to strike a balance between beauty, the way something looks, and usability, the actions a viewer perceives an object capable of performing [14]. This concept is employed to gauge an exhibition or exhibit's potentials to draw attention and to engage the viewer or user. 


\section{EVA 2009 London Conference 6-8 July \\ Francesca Monti}

Finally, concepts and methodologies from visual communication theories were selected to analytically assess an exhibition as a visual object, and to unpack its social, compositional and technological aspects [15]. The aim here is to employ a uniform language to create a description of individual exhibition elements and to trace a correlation between their visual communication qualities and visitor reactions to them.

The following account of the evaluation findings is broadly subdivided according to the three exhibition dimensions. The focus is on the data from the video reconstruction and interactive webpage. The results from the interviews with the curator, interpretation officer and designer are not presented in isolation but included next to other results to offer a comparison between the museum's intentions and visitor reception. The knowledge derived from existing evaluation studies is also introduced as relevant.

\section{THE GALLERY}

Assessment of space

Gallery 61: Ancient Egyptian life and death, the tomb-chapel of Nebamun (figure 3) is on the upper floor of the British Museum. It is aligned on the eastern-western axis on which six adjacent corridor rooms trace the development of ancient Egyptian culture. It can be accessed from the Egyptian Death and Afterlife Gallery, and from the West stairs.

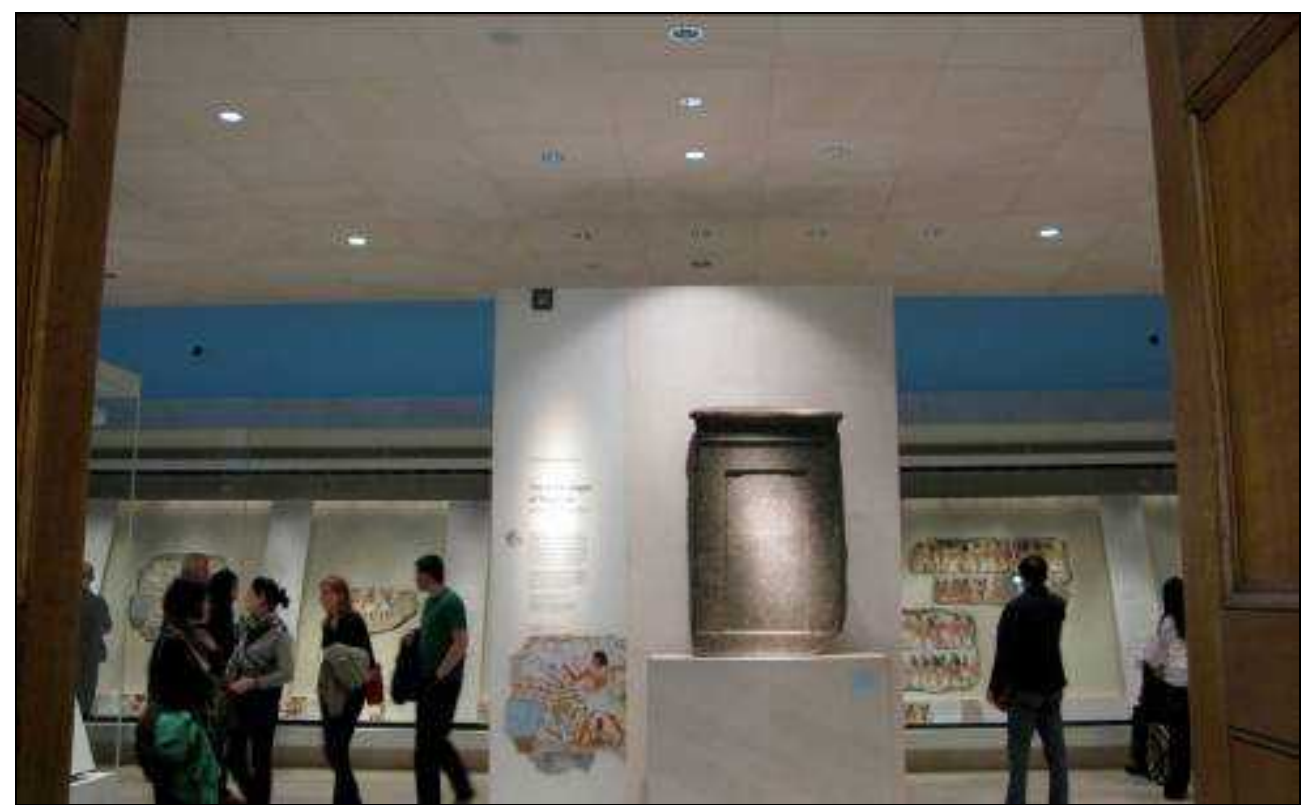

Figure 3. View of Nebamun tomb-chapel at the British Museum from the West stairs.

The exhibition is designed to resemble the layout of a tomb-chapel (figure 3 and 4). The walls and false ceiling of the gallery are of limestone to match the colour of the Theban hills where the Nebamun burial chamber is now lost; the walls are sky blue, as if under the Egyptian sun. The room primarily hosts eleven beautiful paintings from the ancient Egyptian tomb-chapel of Nebamun (approximately 1350 BC) depicting, for example, vivid scenes of Nebamun as a tall young man fowling in the marshes, stacks 
of food offerings, and naked dancing girls. These are impressively presented in a showcase on the northern wall, and in an adjacent free-standing display. The themes in the paintings (living conditions, work and survival, beauty, fashion and offerings from a tomb) are three-dimensionally brought to life by 180 objects from the same period; some of these are visually spectacular, others inconspicuous.

Although each display can be viewed in isolation, the keen visitor will be able to understand at least the primary level of conceptual organisation. From both access points the viewer has an overview of the whole exhibition, and an uninterrupted vista over the impressive display of paintings. But only a closer inspection reveals the existence of the video in a segregated area on the western end of the space. When entering from the southern stairs one immediately encounters the gallery's introductory panel next to a large stela (figure 3).

Interpretative material, such as large photographs of Egypt, drawings, and a 3D reconstruction of the tomb-chapel show how the structure would have been in its original setting and pristine conditions.

The interpretation strategy adopted for Gallery 61 is a multileveled one. The museum's overall intention was to geographically and culturally contextualise the pictorial fragments to offer an experience as close as possible to walking into the original chapel $[16,17]$ and to offer a glimpse of life in ancient Egypt [18] by showing the differences between the rich and the poor [17]. At the same time, the paintings and ancillary objects are employed to illustrate that the ancient Egyptian were as sophisticated as we are; to convey the extraordinary artistic and aesthetic merits of the paintings; and to invite a reflection on the lack of evidence, and on how curatorial knowledge is restricted to the material evidence currently available [16]. It is accepted that individual visitors may be reached by different facets of the messages; they are invited to take whatever they are ready for on the day [ibid.].

A study of the spatial configuration of the gallery and of the rapport between individual exhibits supports the curator's belief that the successful deliverance of the messages is predominantly due to the visual design and to the carefully planned lay out of exhibits [ibid.]. For example, items usually present in funerary scenes are displayed next to the painting depicting a table of offerings (Figure 4, items 16 and 17). Observations of visitor behaviour confirmed that some individuals look at the two displays in combination. Evidence from the assessment of the reconstructive video also shows that the digital medium is central to the geographical and architectural contextualisation of the paintings and to the corroboration of the synergic relationship between objects and paintings. 


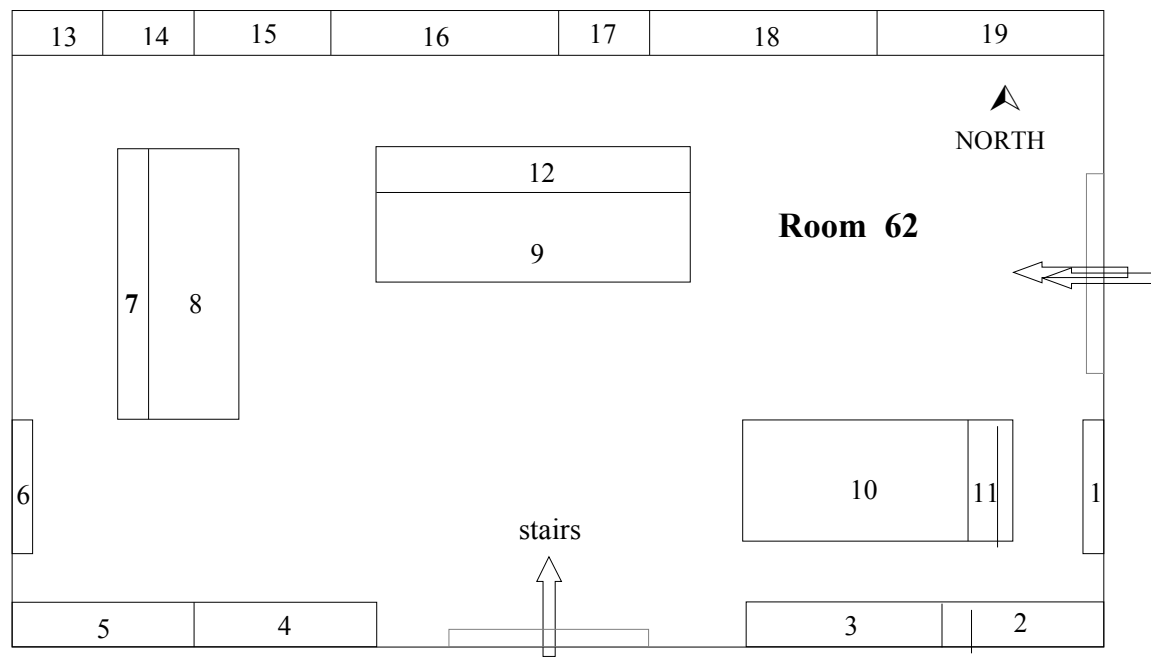

1. Living in Ancient Egypt

2. Leisure

3. Fashion and Beauty

4. Living Conditions

5. Work and Survival

6. Living in Ancient Egypt

7. AV

8. Painting: Surveying the fields

9. Introduction and stela

10. Furniture

11. The Wealthy Home

12. Tomb Building Tools

13. Painting: Nebamun Gardens

14. Painting: Nebamun Hunting in the Marshes

15. Painting: Offering Bringers

16. Painting: Offerings

17. Statues and Offerings

18. Painting: Feast

19. Painting: Geese and Cattle

Figure 4. Schematic plan of Nebamun Gallery.

Tracking and observation exercises carried out by the British Museum [9] revealed the attracting and holding power of individual displays (figure 4). Attracting power is about the number of people that stop to look at an exhibit, while holding power refers to the amount of time visitors interact with an exhibit. Overall visitors spend on average 442 seconds in Gallery 61; this is exceptionally high in comparison to other galleries. The work and survival display had the highest attracting power, followed by the painting depicting Nebamun in the marshes and the feast scene (figure 5). The high attracting rate of the humble-looking objects within the work and survival display may be due to their location in relation to a recorded high visitor tendency to turn left when entering from the West stairs.

The degree of engagement with the objects was also overall high, with a minimum recorded rate of at lest $60 \%$ of visitors who spent between 10-40 seconds or more with an exhibit [9]. Crucially, despite a segregated location the video recorded the highest level of interaction: over $70 \%$ of visitors used it, $97 \%$ had a meaningful engagement with it and most of them stayed for its entire duration [ibid.].

\section{Assessment of the video by means of check-tables}

The presence of the three-dimensional computer reconstruction of how the tombchapel may have looked like (figure 5) is significant because it is one of the first of this kind in a permanent gallery at the British Museum and constitutes a departure from the museum's traditional modes of interpretation. The proposal to include the AV in the gallery was initially received with trepidation by the curator, who feared that the video might distract from the objects [16]. However, the exhibit's great potentials to 


\section{EVA 2009 London Conference 6-8 July \\ Francesca Monti}

contextualise the paintings without the physical need to reconstruct the building was recognised as a significant asset [16,18].

Initial testing with visitors indicated that they were overwhelmingly in favour of its use in the gallery: visitors were attracted to the video, liked it, and thought that it successfully conveyed a sense of the atmosphere within the tomb-chapel [19]. But the formative evaluation also revealed that the museum ought to be clear about the purpose of the resource and unambiguous about the reconstructive process [ibid.]. These findings informed the design of the final version of the digital screen.

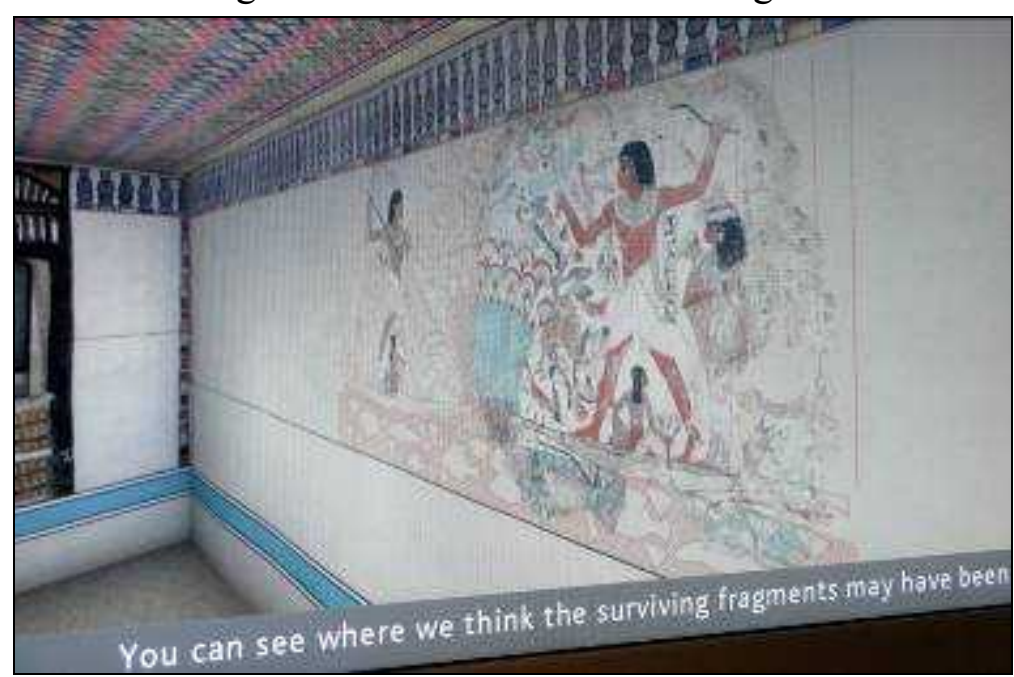

Figure 5. A screenshot from the video reconstruction of Nebamun tomb-chapel.

After having considered a series of factors, such as visitor flow, costs, and aesthetics the museum decided to place the video in a segregated area of the gallery (figure 4) [17]. The placing counteracts the video's potential distracting power, and ensures an unobstructed visitor flow even when large groups of schoolchildren gather around it.

The reconstruction is two minutes long. It consists of a sequence of tree-dimensional frames which take the viewer on a journey of exploration from the desert of Egypt, through the Nile Valley, to the West bank of the river, and into the tomb-chapel. Inside the edifice the eleven paintings are shown in all their beauty, located where they are believed originally to have been (figure 5).

Visitor interaction with the video was assessed using check-tables. This enabled the researcher to take account of the video's design elements, the narrative approach, beauty and usability matters, as well as the communication theories adopted.

Design idioms. The overall design strategy contributes to the successful use of the medium in accordance with the museum's intentions $[16,17,18]$. The video is silent, but each photogram is accompanied by a brief subtitle with explanations about what the viewer sees, the reconstructive process, and a distinction between speculation and evidence-based information. The museum carefully considered the use of accompanying audio, but decided against it, wishing to remain faithful to the contemplative and sombre atmosphere of an Egyptian tomb-chapel [17]. The design of the reconstruction is evocative. The visual and textual information complements the 
gallery objects. The video is helpfully juxtaposed with an introductory panel and series of drawings showing the plan and side elevation of the tomb-chapel. The brief reference to the accompanying interactive webpage on the wall under the screen is not noticeable and would benefit from better signage, as also indicated by visitor accounts. The area is adequately lit, particularly when natural light seeps through the skylight on a bright day.

Object biographies. The video successfully conveys the original physical and sociocultural context of the paintings. By highlighting gaps in knowledge and uncertainty in reconstruction of the tomb-chapel it also invites reflection on the life of the paintings after they left Egypt, when they entered the museum collections. This narrative approach allows the discerning viewer to transcend the materiality of the objects and to consider notions of curatorial knowledge, absence and presence of evidence, and the unavoidable uncertainties underlying archaeological reconstructive processes. The digital exhibit effectively communicates both the visual qualities and the original function of the paintings. Although the reconstruction highlights the beauty and the artistic merits of the fragments, their physicality and purpose also comes across.

Beauty and usability. The video achieves a harmonious effect through design consistency and an adequate and eye-catching use of colour and space. It tantalises the viewer with an evocative contextualisation of the displayed paintings by means of a sequence of dazzling vistas. As well as this successful visual strategy, the video demonstrated a high degree of usability. From the opening frame, which informs as to the duration and subject of the video, there is a clear conceptual and topographical orientation, with each scene explained by means of a succinct caption. The script of the subtitles is legible and the register of language accessible to all. The height at which the video is placed makes it visible to children and wheel-chair users. Its proximity to the tomb-paintings elicits a direct comparison between digital information and real objects.

Visual communication theories. An analysis of the three-dimensional reconstruction by means of a compositional interpretation method [20] elicits a closer inspection of the individual elements of the video: framing, montage, sound, and narrative structure. Each individual photogram is clearly and eloquently framed, with no unnecessary clutter of visual information and so focusing the viewer's attention on the intended key messages. Individual shots are seamlessly and coherently put together. The choice of not including any sound achieves a closer focus on the visual qualities of the exhibit. Overall the first level of the story, concerned with a contextual reconstruction of the paintings, comes across clearly. Other levels of the narrative, such as the theme of evidence-derived curatorial knowledge, are accessible to more discerning users.

\section{Assessment of the webpage by five criteria}

The gallery's computer simulation of the tomb-chapel is accompanied by an interactive version available at the museum's website [21]. The online rendering allows the user to explore different rooms of the tomb-chapel, to gain additional information about them, and to translate the hieroglyphic inscriptions from the paintings.

The digital resource was evaluated by means of five criteria [8]: how easy it was to find the interactive animation within the museum homepage; the overall design (colour, text, size, layout); how easy it was to navigate within the resource; the extent and type 
of information provided; and whether the tool was representative and in tune with the interpretation strategy of Gallery 61.

Easy to find. The interactive video did not rate high on this criterion. The digital resource, which is available from the webpage of Gallery 61, is not easily accessible from the museum homepage; there is no direct reference to it there. The simplest way to find the virtual tomb-chapel is through a free text box search on the homepage. Alternatively, it can be accessed via the explore tab on the homepage, and then navigating through the galleries sub-section to Gallery 61. Both search paths require prior knowledge of the existence of the resource, awareness of the related gallery, and the user's resolution to find it.

Good design. Once located the digital tool the user is rewarded with an intuitive to use and pleasantly designed reconstruction of the ancient tomb-chapel. The pagination is well designed and effectively delivered, with a good ratio between empty space and information, which result in an uncluttered and pleasant virtual environment. Both textual and visual content are presented logically and sequentially. The colour scheme is appropriately employed to render key architectural and pictorial details. The amount of text and the size of the font are satisfactory, and the accompanying explanations informative and without unnecessary jargon, its register suitable to a broad audience.

User-friendliness. The resource is intuitive to use and can be easily navigated even by an inexperienced virtual visitor. The journey within the tomb-chapel is conceptually and spatially subdivided in six parts, each one corresponding to a tomb-chapel's room. The navigable screen is split in two parts: the larger portion on the right with the threedimensional rendering of the space, and on the left a list of six links corresponding to the tomb's rooms. This interactive directory is accompanied by a small plan of the tomb-chapel, which provides an overview of the architecture and offers feedback as to the user's whereabouts within the building. A simple navigation bar facilitates a 360 degrees tour and zooming in and out of the space. A large interactive arrow on the floor suggests potential paths to adjacent rooms. An hand-shaped icon next to the paintings alerts the user of the existence of hotspots and of the possibility to gain additional information. It is easy to return to a previous screen or to start again from the courtyard either by clicking on the desired location on the tomb's plan, by selecting a different link from the list on the left, or by choosing the back button.

Type of information per item. The interactive reconstruction comprises a wealth of visual and textual information and references to the museum displays in Nebamun Gallery. Navigable spaces can be viewed from different perspectives, details of the tomb-chapel are available for closer inspections by zooming in and out, selected items are explained by means of additional text, and a translation is offered for the Egyptian inscriptions. The homepage for Gallery 61 also provides an exploration of the conceptual themes presented in different displays of the exhibition, and shows textual information and photographs for a selection of objects from the exhibition.

Representativeness of the interpretation strategy. The virtual reconstruction successfully conveys most themes at the core of the museum's interpretation strategy. The connection between virtual and physical objects established through the introductory webpage, together with the gallery's video and the meticulous layout and 
design of the gallery, contributes to linking all the elements of the exhibition. The virtual environment effectively and atmospherically communicates the sophistication of the people who created the paintings, the aesthetic merits of the depictions, and proposes a reflection over the limitations of any evidence-based knowledge.

\section{THE VISITORS}

\section{Survey}

Twenty-five people were interviewed for the purpose of this study. Sixteen of these were female and nine male. The age ranges of the interviewees are fairly distributed across the groups, with a slight predominance of visitors between the age of 45 and 54 . Fourteen out of twenty-five visitors were visiting the museum with a partner of friend, six with family, and five alone. In line with the results of the British Museum's own survey [9] a considerable portion of the sample (seven out of twenty-five respondents) came to museum specifically to visit Nebamun Gallery on the wave of the advertising for its opening in January 2009. This marketing campaign may somehow have impacted on the results of the evaluations [ibid.] and on visitors' perception, reception, and recollections of the exhibition.

Twelve out of twenty-five visitors watched the 3D reconstruction. The most common cause for not looking at the video was not being aware of its existence (ten responses) followed by time limitations (three responses). Although the segregated placing of the digital exhibit was a deliberate museum strategy, its hidden location deprives some visitors from the choice to view it. This issue needs consideration, as visitors should have the option to decide whether to interact with the exhibit or reject it. The presence of the screen could be signalled by means of a discrete sign or mentioned in the gallery's introductory panel which observation shows to receive a high degree of visitor attention [9].

The contextualising ability of the video and its contribution to the deliverance of the gallery's messages emerged from the findings. Fifteen examples of what visitors particularly liked in the digital reconstruction were recorded. Visitors' favourite aspects are the video's ability to deliver the atmosphere of the original setting (five out of fifteen) and to convey a sense of the past through the full colour reconstruction of the tomb-chapel (five out of fifteen). Further proof of the role of the digital resource in the meaning-making process came from the replies to the question: "How did you relate what you saw in the animation to the objects in this gallery?". According to six out of sixteen interviewees the most valuable aspect of the video is its ability to place the paintings in their original cultural, geographical and architectural context. Significantly, four mentions were made of the reconstruction's role in offering a visual and straightforward representation of the relationship between the amount of paintings preserved and what is now lost. It is very significant that this higher-level message, which relates to the limitations of object-derived knowledge and the representation of the past in academic institutions, was recognised and highlighted by visitors.

Overall, six out of ten viewers thought that the video enhanced their understanding of the objects in the gallery. Two visitors who believed the contrary went on to explain that most of the information conveyed by the digital reconstruction already came across 
from other exhibits. This result can be looked in a positive light and interpreted as an indication of the video's ability to visually corroborate some of the gallery's themes.

Finally, twenty interviewees were in favour to the potential introduction of other similar digital resources in the museum galleries. In particular four visitors commented on the appropriateness of three-dimensional reconstructions to convey a sense of the original archaeological settings, and two thought they can be an apt medium to contextualize objects taken away from their countries of origin. Five respondents, however, added the caveat that they would welcome the introduction of other screens only if they were employed in moderation, if they added to the narrative, and if they followed consultation with audiences.

\section{CONCLUSIONS}

The methodology employed in this study constitutes an effective basis for a comprehensive assessment of an exhibition and its individual elements. The conceptual framework on which it was built facilitated a multilayered analysis of the visitor encounter with the digital resources, taking account of the spatial syntax of where the encounter takes place; the characteristics of objects and their presentation within the gallery; the viewer's emotional, aesthetic, affective and cognitive reactions to the exhibits; and his or her interplay with the surrounding environment. This conceptual and methodological framework can be expanded to provide a full-scale evaluation of the gallery-museum-visitor dimension.

In the tomb-chapel of Nebamun the dilemma of how to display inconspicuous and spectacular objects side-to-side avoiding viewing disparities is successfully tackled. Each interpretative item, each object, is a piece in a wider picture of the deceased's world. Thus, even in a space where the spectacular could have easily dominated, a fair distribution of attention is achieved; visitors compare different sources of evidence and draw connections between the physical and the pictorial, the textual and the digital. The viewer is simultaneously storyteller and spectator, and the stories are conveyed by different media. Because each exhibit is necessary to the deliverance of the gallery's narratives visitors are enticed to make a visual journey and an investigation of different sources, which gradually reward them with additional pieces of the broader picture. This is achieved through a carefully planned layout, an effective design and interpretation strategy, an appropriate ratio between space and objects, and the recreation of an atmospheric and evocative place. There is little distortion between the museum communication intentions and visitors' reception of them.

In this scenario the three-dimensional reconstruction plays a crucial unifying and contextualising role. Although the exhibit's concealed location could be advertised more clearly, visitors who find the screen are rewarded with a clear, effective and atmospheric means to contextualise the paintings and an opportunity to reflect over the messages at the core of the interpretation strategy. After the visit the zealous visitor can plunge into an additional web-based virtual exploration of the ancient Egyptian monument to reach deeper and more detailed levels of understanding and to savour, once again, the evocative and moving atmosphere of Nebamun Gallery. 


\section{ACKNOWLEDGEMENTS}

I am indebted to the British Museum for allowing me to conduct this study of Nebamun Gallery. In particular, my gratitude goes to Xerxes Mazda, Richard

Parkinson, Geoff Pickup, and Rebecca Richards for sharing their experience with me.

\section{References}

[1] MONTI, F. Allowing objects to speak, people to hear : the effective display of inconspicuous objects from Egyptian collections. 2007. [Unpublished doctoral thesis submitted to the University of London.]

[2] CSIKSZENTMIHALYI, M. and HERMANSON, K. Intrinsic motivation in museums : what makes visitors want to learn? Museum News. 74, 1995. pp.3537.

[3] MORPHY, H. Aesthetics in a cross-cultural perspective : some reflections on Native American basketry. Journal of the Anthropological Society of Oxford. 23, 1992. pp.1-16.

[4] CSIKSZENTMIHALYI, M. Why we need things. In : S Lubar and WD Kingery (eds.) History from things : essays on material culture. Washington and London : Smithsonian Institution Press, 1993. pp.20-29.

[5] SHETTEL, H.H. Exhibits : art form or educational medium? Museum News, 51, 1973. pp.32-41.

[6] ALT, M.B. and SHAW, K.M. Characteristics of ideal museum exhibits. British Journal of Psychology. 75,1984. pp.25-36.

[7] BITGOOD, S. An analysis of visitor circulation : movement patterns and the general value principle. Curator. 49, 2006. pp.463-475.

[8] MONTI, F. Digital issues. In: S. Keene. Collections for people : museums' stored collections as a public resource. London : UCL, 2008.

[9] MINER, R. Evaluation of Gallery 61 Ancient Egyptian Life and Death : The Tomb Chapel of Nebamun (1350 BC). 2009. [Unpublished internal document from the British Museum Department of Learning and Audiences.]

[10] HILLIER, B. and HANSON, J. The social logic of space. Cambridge : Cambridge University Press, 1984.

[11] HALL, M. On display : a design grammar for museum exhibitors. London : Lund Humphries, 1987.

[12] APPADURAI, A. (ed) The social life of things : commodities in cultural perspective. Cambridge : Cambridge University Press, 1986.

[13] ALBERTI, S.J.M.M. Objects and the museum, Isis. 96, 2005. pp.559-571. http://www.journals.uchicago.edu/ISIS/journal/issues/v96n4/960404/960404.web.pdf [Accessed 15 May 2009.]

[14] NORMAN, D.A.Emotional design: why we love (or hate) everyday things. New York : Basic Books, 2004.

[15] ROSE, G. Visual methodologies. London : SAGE publications, 2001.

[16] PARKINSON, R. [Unpublished interview with the curator conducted for the evaluation of Gallery 61.] 2009. 
EVA 2009 London Conference 6-8 July

Francesca Monti

[17] RICHARDS, R. [Unpublished interview with the interpretation officer conducted for the evaluation of Gallery 61.] 2009.

[18] PICKUP, G. [Unpublished interview with the designer conducted for the evaluation of Gallery 61.] 2009.

[19] WILEY, K. Nebamun AV testing. 2009.[Unpublished internal document from the British Museum Department of Learning and Audiences.]

[20] MONACO, J. How to read a film : movies, media and multimedia. London : Oxford University Press, 2000.

[21] BRITISH MUSEUM. The tomb-chapel of Nebamun interactive webpage. http://www.britishmuseum.org/explore/galleries/ancient_egypt/room_61_tombchapel nebamun.aspx [Accessed 24 May 2009.] 